\title{
In vivo and in situ characterization of leaves using OPC technique
}

\author{
A.C. Pereira*, J.J. Alvarado-Gil**, O. Zelaya**, H. Vargas and N. Cella*** \\ Instituto de Física Gleb Wataghin, Universidade Estadual de Campinas, UNICAMP, 13083-970 Campinas, \\ São Paulo, Brazil \\ * Departamento de Física, Universidade Federal do Maranhão, 65080-040 São Luis, MA, Brazil \\ ** Centro de Investigación y Estudios Avanzados, INP, A.P. 14-740, México 07000, Mexico \\ *** Instituto Politécnico, Universidade do Estado do Rio de Janeiro, 28601-970 Nova Friburgo, RJ, Brazil
}

\begin{abstract}
In this paper the use of the so-called open photoacoustic cell (OPC) detector technique for in vivo and in situ measurements is described. Spectroscopic and photosynthetic properties in undetached maize leaves are measured. Photosynthetic oxygen evolution detected by OPC apparatus is estimated using a Clark-type oxygen electrode sensor.
\end{abstract}

\section{INTRODUCTION}

Photoacoustic spectroscopy (PA) and related photothermal techniques $[1,2]$ offer an arsenal of different configurations of sensors and experimental arrangements, for study of optical and thermal properties of materials. Recently, open photoacoustic cell (OPC) was suggested as an alternative photoacoustic method $[3,4]$. In the OPC configuration the sample is placed atop a commercial electret microphone and, unlike conventional PA, no additional transducer medium is required. Front air chamber of electret microphone is used as a transducer medium itself. The proposed OPC technique is particularly suitable for in vivo studies in biological applications.

During last ten years conventional PA was applied in the area of plant photosynthesis research $[5,6]$. Usually, studies are carried out with isolated chloroplasts or pieces of (even whole) detached leaves. In contrast to this method, the OPC technique was shown capable of conducting in vivo measurements with intact undetached leaves [4,7]. In this paper, sensitivity and feasibility of OPC method for monitoring spectroscopy and photosynthetic properties of leaves using normal green, striped and albino maize leaves were evaluated.

\section{EXPERIMENTAL PROCEDURE}

\subsection{OPC detector}

Figure 1 shows a commercial electret microphone (and its cross section) operating as a OPC detector. The front sound inlet is a circular hole ( $3 \mathrm{~mm}$ diameter). The front air chamber adjacent to the metallized face of diaphragm has a diameter of $7 \mathrm{~mm}$ and is roughly $1 \mathrm{~mm}$ long. Additional details can be found elsewhere $[3,4]$.
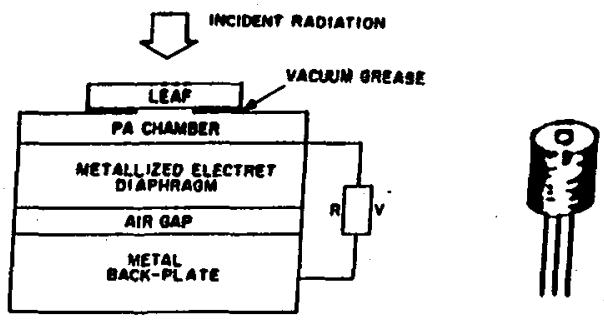

Figure I - A commercial electret microphone and the cross section of the OPC apparatus.

\section{$2.2 \mathrm{O}_{2}$ electrode detector}

The cross section of $\mathrm{O}_{2}$ electrode sensor is shown in Fig. 2. The detector is an acrylic cylinder with the oxygen detector inserted, leaving a column of air $\left(0.58 \mathrm{~cm}^{3}\right)$ between the electrode membrane 
and leaf sample. The hole on top of the cell has a diameter of $3 \mathrm{~mm}$, similar to the OPC detector. The electrode used was of Clark-type $\mathrm{Pt} / \mathrm{Ag} / \mathrm{AgCl}_{2}$ and was manufactured by Radelkis. Two thin tubes were connected to the sensor chamber to enable its calibration [8].

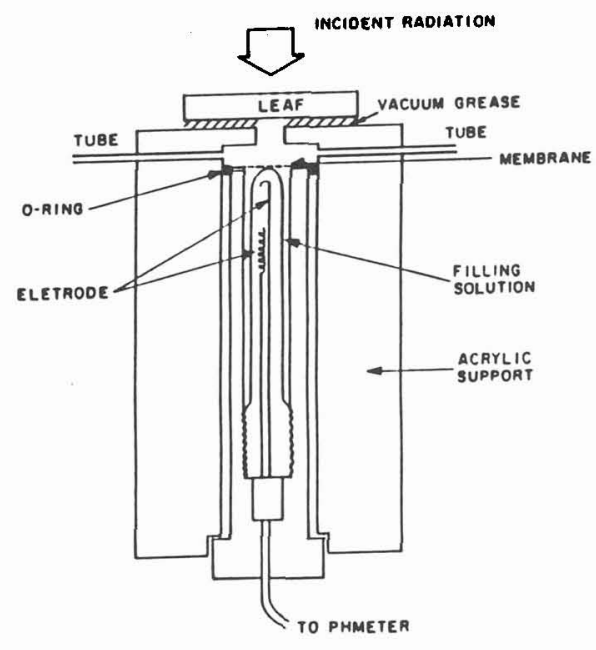

Figure 2 - Schematic cross section of Clark-type $\mathrm{O}_{2}$ electrode detector.

\subsection{Plant materials}

Three different maize leaves were used in measurements. Figure 3 shows schematically the normal green leaf and the chlorophyll-deficient mutants (striped and albino) [9]. In both experiments (e.g., OPC and $\mathrm{O}_{2}$ electrode detectors), the leaf was held flush against the sensor by means of a vacuum grease.

\subsection{Experimental arrangement}

The OPC experimental arrangement is schematically shown in Fig. 4. As described elsewhere [7,9], it consists basically of a modulated monochromatic excitation light beam and a strong dc white light to saturate photosynthesis $[5,6]$. For a Clark-type $\mathrm{O}_{2}$ electrode measurements, the same arrangement was used, with exception of white light and, of course, the detector. The OPC operation for in vivo and in situ measurements is schematically shown in Fig. 5. Again, for $\mathrm{O}_{2}$ electrode measurements, the same procedure was used, except for the white light.

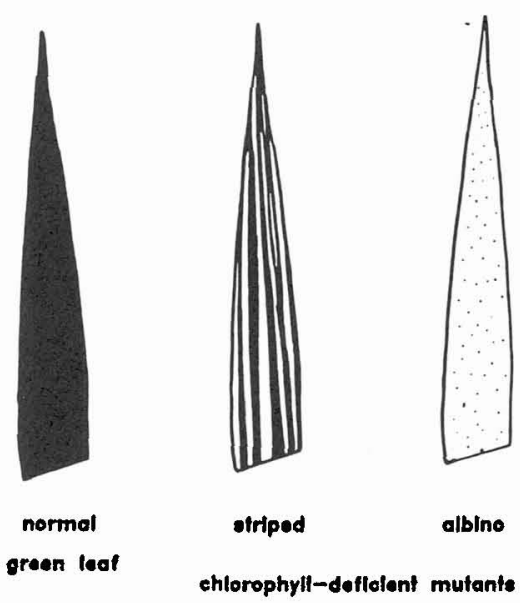

Figure 3 - Three different types of maize leaves used in experiments.

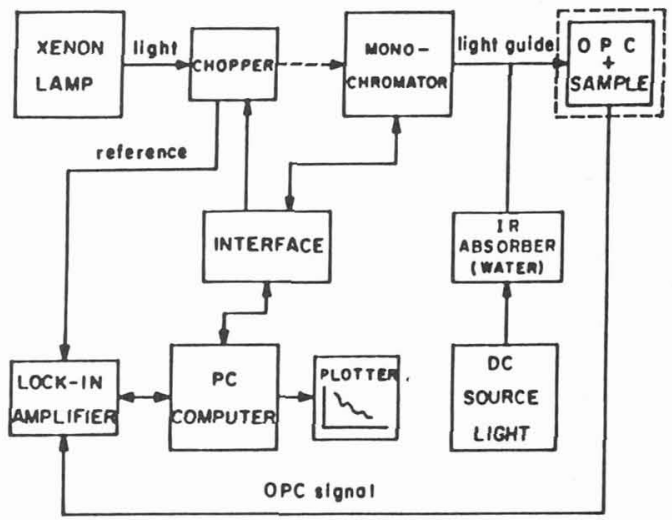

Figure + - Block diagram of the experimental arrangement.

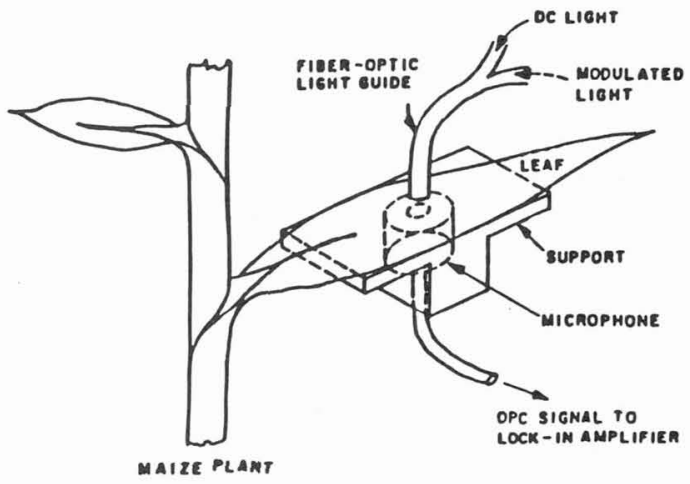

Figure 5 - Exploded view of the OPC procedure for in vivo and in situ measurements. 


\section{RESULTS AND DISCUSSION}

The first measurements carried out were those to spectroscopically characterize three different maize leaves. Different proportions of green and chlorophyll-deficient areas were chosen by adjusting the $3 \mathrm{~mm}$ diameter front sound inlet of the OPC detector over the desired leaf area. As described elsewhere [4], in case of optically transparent sample, the OPC signal has two main photothermal sources. One, coming from the sample absorption (as in conventional PA), and another one due to absorption of transmitted light (see Fig. 1) by metallized diaphragm. The absorption spectra, shown in Fig. 6, were obtained by taking into account these contributions [4]. During the acquisition of spectrum the background white light was turned on to avoid the presence of photobaric component [5] in the OPC signal. As expected, differences in absorption spectra were observed (Fig. 6), showing that the OPC method possess a resolution high enough to assess in vivo and in situ low levels of chlorophyll and carotenoid in leaves of chlorophyil-deficient mutants.

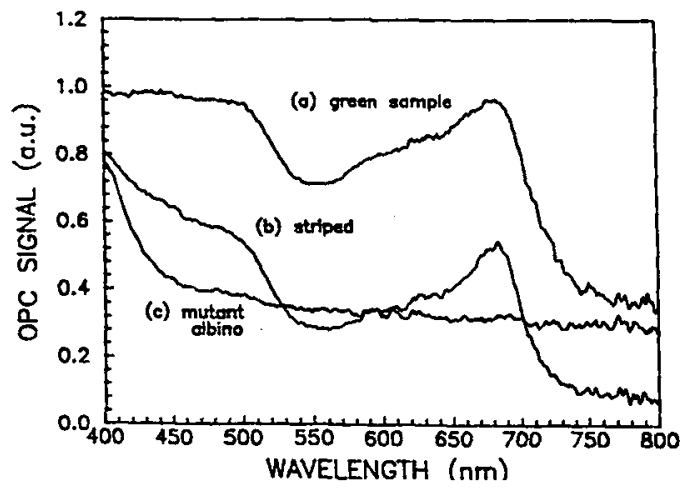

Figure 6 - Normalized photoacoustic spectra [4] of undetached maize leaves: normal green leaf (a); approximately $50 \%$ of green sector on a striped leaf (b) and albino leaf (c). The intensity of comtimuous background white light was $200 \mathrm{~W} . \mathrm{m}^{-2}$ and modulation frequency used was $17 \mathrm{~Hz}$.

Using the same procedure to choose different proportion of green and chlorophyll-deficient areas in different maize leaves, experiments were carried out to detect oxygen evolution. As in conventional PA [6], the OPC signal has the photobaric contribution, resulting from periodic oxygen evolution accompanying the modulated photosynthesis [7]. The modulated oxygen flow into the OPC chamber (or even in intercellular spaces inside of the leaf), produced during photosynthesis, induces a pressure fluctuation in the OPC chamber. For a given modulation frequency, in photosynthetic steady state, the photothermal contribution (sample + diaphragm membrane) for the OPC signal, is constant in time even when the background, non-modulated light, is turned on. On the other hand, the photobaric contribution can be eliminated by the background non-modulated light $[6,7]$. As the saturating background light induces a continuous photosynthesis, modulated excitation light does not affect photosynthetic process and hence no modulated oxygen is produced. It results in an amplitude decrease of OPC signal (negative effect) and by an increase of photothermal component (positive effect). At low modulation frequency, the net effect is negative. As the modulation frequency increase, the net effect becomes smaller and eventually becomes positive. The oxygen evolution measurement is based on the negative effect amplitude of the OPC signal.

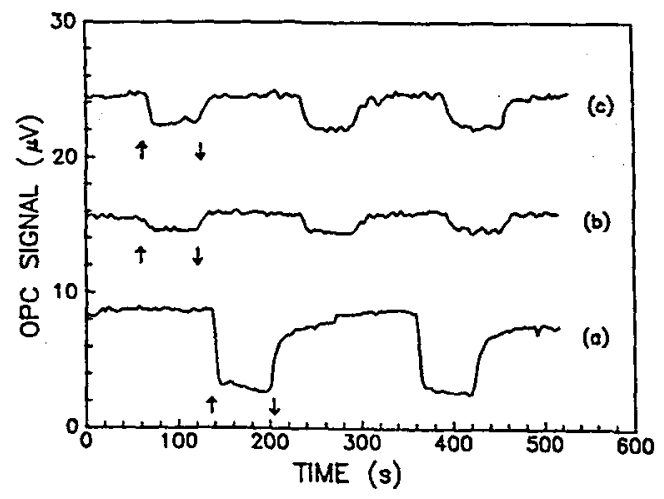

Figure 7 - OPC measurements of negative effect in undetached maize leaves: normal green leaf (a); approximately $20 \%$ of green sector on a striped leaf (b) and albino leaf (c). The intensity of the modulated light at $680 \mathrm{~nm}$ uas $16 \mathrm{~W} \cdot \mathrm{m}^{-2}$ and that of contimuous background white light $(\uparrow$ on, $\downarrow$ off was $200 \mathrm{~W} \cdot \mathrm{m}^{-2}$. The modulation frequency was 17 $\mathrm{Hz}$.

Figure 7 shows the amplitude of OPC signal plotted versus time at $17 \mathrm{~Hz}$ frequency modulation for three types of maize leaves. In case of striped mutant type just a $20 \%$ of green area of a total detected area was chosen. It is shown that even in albino mutant type oxygen evolution can be observed by the OPC method. It is interesting to 
observe that the total OPC signal is higher for albino mutant type, suggesting higher membrane contribution for the photothermal component. More details on these results can be found in Ref. 9.

In order to quantify the oxygen evolved, a correlation between the oxygen measured by the OPC method (negative effect) and the oxygen measured by a Clark-type $\mathrm{O}_{2}$ electrode (details can be found elsewhere [8]) was made. Using the same experimental arrangement as in the OPC case, the $\mathrm{O}_{2}$ electrode sensor was employed to measure $\mathrm{O}_{2}$ evolved under the same light, frequency and leaf experimental conditions. Care was taken to ensure that the same quantity of chlorophyll pigments was present in both sensors. This was done by choosing two adjacent areas (about $5 \mathrm{~mm}$ distant), just avoiding the remaining vacuum grease. OPC measurements were done before positioning the leaf on the $\mathrm{O}_{2}$ electrode detector. In such correlation experiment, a normal green leaf with three different excitation light power was used. Figure 8 shows the correlation between both type of oxygen sensors. The small deviation from zero at the origin can be attributed to several parameters. One of them is the fact that $\mathrm{O}_{2}$ electrode sensor was operated near its limit. Another one is related to the positive effect and light saturation. However, what is important to know is that exist a correlation between both measurements enabling some kind of calibration.

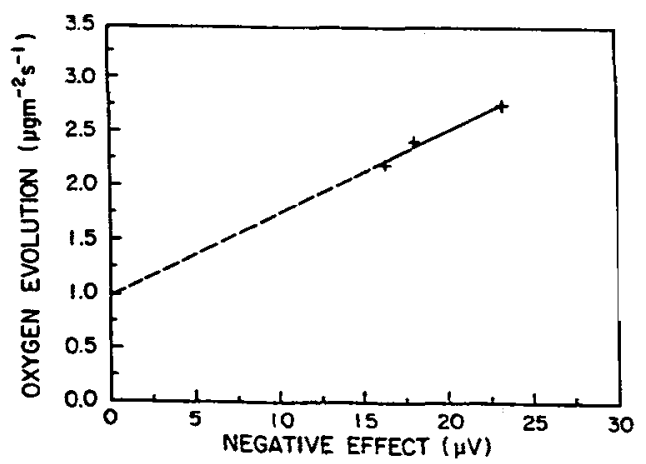

Figure 8 - Correlation between signal from $\mathrm{O}_{2}$ sensor and the $O P C$ signal. Three different maize plants were studied using three different levels of modulated light intensity at $680 \mathrm{~nm}$.

Assuming once the above correlation (taking the angular coeficient of its linear regression), each unit of OPC signal for the negative effect correspond to
$0.08 \mu \mathrm{g} \cdot \mathrm{m}^{-2} \cdot \mathrm{s}^{-1}$ of oxygen evolution. Therefore, using a striped mutant maize leaf, several proportions of green area (to the total area) were chosen and the corresponding negative effect obtained. After that, these negative effects were quantified by the correlation and the green areas were estimated by using a stereomicroscope with a scaled objective; Table 1 gives these results.

Table 1 - Negative effect and oxygen evolution from a striped mutant maize leaf.

\begin{tabular}{|c|c|c|}
\hline $\begin{array}{c}\text { Green area } \\
(\%)\end{array}$ & $\begin{array}{c}\text { Negative effect } \\
(\mu \mathrm{V})\end{array}$ & $\begin{array}{c}\mathrm{O}_{2} \text { evolution } \\
\left(\mu \mathrm{g} \cdot \mathrm{m}^{-2} \cdot \mathrm{s}^{-1}\right)\end{array}$ \\
\hline 30 & 2.0 & 0.16 \\
\hline 50 & 3.9 & 0.31 \\
\hline 75 & 5.6 & 0.45 \\
\hline 100 & 11.4 & 0.91 \\
\hline
\end{tabular}

In conclusion, feasibility of OPC technique was demonstrated as a simple and reliable method for in vivo and in situ studies of photosynthesis using intact undetached leaves.

We acknowledge support of CNPq, CAPES and FAPESP.

\section{REFERENCES}

[1] Rosencwaig A., Photaocoustics and Photoacoustic Spectroscopy (New York: Wiley, 1980).

[2] Vargas H. and Miranda L.C.M., Phys. Rep. 161 (1988) 45-101.

[3] Silva M.D., Bandeira I.N and Miranda L.C.M., J. Phys. E: Sci. Instrum. 20 (1987) 1476-1478.

[4] Marquezini M.V., Cella N., Mansanares A.M., Vargas H. and Miranda L.C.M., Meas. Sci. Technol. 2 (1991) 396-401.

[5] Bults G., Horwitz B.A., Malkin S. and Cahen D., Biochim. Biophys. Acta 679 (1982) 452456.

[6] Poulet P., Cahen D. and Malkin S., Biochim. Biophys. Acta 724 (1983) 433-446.

[7] Pereira A.C., Zerbetto M., Silva G.C., Vargas H., Silva W.J., Oliveira Neto G., Cella N. and Miranda L.C.M., Meas. Sci. Technol. 3 (1992) 931-934.

[8] Pereira A.C., Oliveira Neto G., Vargas H., Cella N. and Miranda L.C.M. (to appear in Rev. Sci. Instrum.)

[9] Pereira A.C., Prioli L.M., Silva W.J., Oliviera Neto G., Vargas H., Cella N. and Alvarado-Gil J.J., (to appear in Plant Science) 